\title{
Quantum Molecular Dynamics calculations of radiative opacities
}

\author{
S. Mazevet, L. A. Collins, N. H. Magee, J. D. Kress, and J. J. Keady \\ Theoretical Division, Los Alamos National Laboratory, Los Alamos, NM 87545, USA \\ Received 11 April 2003 / Accepted 7 May 2003

\begin{abstract}
We show that Quantum Molecular Dynamics provides a powerful tool to extend and benchmark current opacities libraries into the complex regime of warm dense matter. In this regime, the medium can be constituted of electrons, protons, atoms and molecules, while plasma and many-body effects can not be treated as perturbations. Among the most notable features of this new approach for calculating Rosseland mean opacities is the ability to obtain a consistent set of material (equation-ofstate), optical and electrical properties for various mixtures from the same simulation.
\end{abstract}

\begin{abstract}
Key words. atomic processes - dense matter - equation of state - plasmas - radiation mechanisms: general planets and satellites: general
\end{abstract}

\section{Introduction}

The modeling of the environments found in a diverse range of astrophysical and terrestrial locales requires accurate radiative opacities and their associated equations-of state (EOS). A few examples such as planetary and stellar atmospheres, inertial confinement fusion, shock-compressed fluids, and high-energy density plasmas amply demonstrate the scope of applications. For systems composed of dilute gases or plasmas, standard libraries, based on essentially isolated atoms (Rickert 1995), provide reasonable representations of the attenuation of radiation through the medium. Their construction involves the collation of individual vacuum atomic processes such as freefree, bound-free, and bound-bound transitions. These libraries have served well as the foundations of large-scale radiationhydrodynamics programs that model many phenomena.

However, for some classes of systems, the density reaches such an extent that interactions among the surrounding particles begin to influence the transitions at a given atomic site. In this case, the incorporation of density effects becomes essential to adjust properly the microscopic properties. If the interactions remain weak then perturbative approaches (Iglesias et al. 2002; Perrot 1996; Crowley \& Harris 2001; Seaton et al. 1994) provide a reasonable approximation to collective effects and can extend the basic libraries into moderately dense regimes. For increasing densities, the interactions among various species no longer stay perturbative and a more global, egalitarian approach must prevail. In addition, as the interparticle separation narrows, the electronic wavefunctions at different sites overlap more strongly, and many-electron quantum mechanical aspects must enter. Such nonperturbative density effects may play

Send offprint requests to: S. Mazevet, e-mail: sdm@t4 . lanl.gov important roles, for example, in the structure of giant and extrasolar planets (Chabrier \& Baraffe 2000) and in the cooling rates of white dwarfs, especially determining their efficacy as astrochronometers (Fontaine et al. 2001).

Quantum molecular dynamics (QMD) offers an effective approach in which all particles receive an equal, global treatment (Collins et al. 2001). For the regimes considered here, an effective separation of the nuclear and electronic motions becomes viable in which a classical treatment applies to the nuclei and a quantum mechanical one to the electrons. The method temporally evolves a representative collection of $\mathrm{N}$ atoms by performing a fully quantum mechanical electronic-structure calculation of the energy, forces, and wavefunctions at each time step. These basic quantities determine a consistent set of static (pressure and internal energy), dynamical (diffusion, viscosity, and thermal conductivity), and optical (electrical conductivity) properties. The approach has shown considerable versatility in applications to solids, plasmas, fluids, and gases and in representations of the transitions between and mixtures of these states. For example, the electrical conductivity determined by QMD (Desjarlais et al. 2002) for aluminum agrees well with imploding-wire experimental findings for a range in environments from solid metal to gaseous plasma. In addition, EOS and conductivity results (Mazevet et al. 2002, 2003; Kress et al. 1999) generally follow gas-gun and high-energy-density experiments for a wide variety species and conditions or, when disagreements arise, serve to delineate the underlying physical processes and constituents. While computationally intensive, the method seems ideal for benchmarking and systematically improving the density corrections introduced within present opacity libraries as well as for providing a solid basis for extending opacity calculations into high-density, stronglycoupled, multi-component environments. 


\section{Formalism}

One common representation of the radiative opacity follows from the Rosseland mean (Perrot 1996) given by

$\frac{1}{\kappa_{R}(\rho, T)}=\frac{\int_{0}^{\infty} \mathrm{d} \omega n^{2}(\omega) B^{\prime}(\omega, T) \alpha(\omega)^{-1}}{\int_{0}^{\infty} \mathrm{d} \omega n^{2}(\omega) B(\omega, T)}$,

where the frequency-dependent absorption coefficient $\alpha(\omega)$ and the index of refraction $n(\omega)$ depend implicitly upon the density $\rho$ and temperature $T$ of the medium. $B^{\prime}(\omega, T)$ represents the derivative of the normalized Planck distribution at a given $T$ - a slowly varying function that peaks around $4 k_{\mathrm{B}} T$.

The ability of QMD approaches to determine radiative opacities follows from the fact that the real-part $\sigma_{1}(\omega)$ of the frequency-dependent electrical conductivity $\left[\sigma(\omega)=\sigma_{1}(\omega)+\right.$ $\left.i \sigma_{2}(\omega)\right]$ completely determines the absorption coefficient and the index of refraction (Callaway 1974). We can express $\alpha(\omega)$ in terms of $\sigma_{1}(\omega), n(\omega)$, and the speed of light $c$ as

$\alpha(\omega)=\frac{4 \pi}{n(\omega) c} \sigma_{1}(\omega)$

and the index of refraction in terms of the real $\epsilon_{1}(\omega)$ and imaginary $\epsilon_{2}(\omega)$ parts of the dielectric function $\left[\epsilon(\omega)=\epsilon_{1}(\omega)+\right.$ $\left.i \epsilon_{2}(\omega)\right]$,

$n(\omega)=\sqrt{\frac{1}{2}\left[|\epsilon(\omega)|+\epsilon_{1}(\omega)\right]}$.

The dielectric functions are, in turn, immediately obtained from the electrical conductivity by

$\epsilon_{1}(\omega)=1-\frac{4 \pi}{\omega} \sigma_{2}(\omega)$

$\epsilon_{2}(\omega)=\frac{4 \pi}{\omega} \sigma_{1}(\omega)$

where an application of a Kramers-Kronig relation yields

$\sigma_{2}(\omega)=-\frac{2}{\pi} P \int \frac{\sigma_{1}(y) y}{\left(y^{2}-\omega^{2}\right)} \mathrm{d} y$

$P$ stands for the principal value of the integral. Therefore, knowing only $\sigma_{1}(\omega)$, we can work our way back from Eqs. (6) to (1) to find the mean opacity $\kappa_{R}$.

We employ a well-established representation (Rapport 1995) of such an extended, disordered system as a periodicallyreplicated reference cell of $N$ atoms. For a given configuration of the atoms within this cell at a fixed time, a finite-temperature density-functional (FTDF) calculation in the generalized gradient approximation (GGA) determines the eigenenergies $\epsilon_{i}$ and eigenfunctions $\psi_{i}$ for the active electrons by a diagonalization of the associated Kohn-Sham equations. The FTDF GGA contains static, exchange, and correlation effects. We emphasize that these wavefunctions characterize the electronic charge distribution for the whole system and can represent atomic, molecular, and condensed matter effects. Together with the ionic core interactions, these electronic wavefunctions determine not only the forces on the atomic centers but also the pressure, internal energy, and electrical properties. Using these forces within the classical equations of motion provides an effective molecular dynamics (MD) scheme to translate the atoms to new positions. Repeating the procedure propagates the system in time. From this temporal collection of atomic positions (trajectory), we can also calculate dynamical properties such as diffusion and viscosity. Therefore, unlike many opacity schemes, the QMD provides a consistent set of static, dynamical, and optical properties obtained from a single set of multi-atom periodic wavefunctions.

We then employ these system wavefunctions within the Kubo-Greenwood (KG) formulation (Callaway 1974) to determine the real part of the frequency-dependent conductivity:

$\sigma_{1}(\omega)=\frac{2 \pi}{\Omega \omega} \sum_{i j} F_{i j}\left|D_{i j}\right|^{2} \delta\left(\epsilon_{j}-\epsilon_{i}-\omega\right)$,

where $\Omega$ is the atomic volume, $F_{i j}$ represents an occupation function determined by the difference between the Fermi-Dirac distributions for $\epsilon_{i}$ and $\epsilon_{j}$ at temperature $T$, and $D_{i j}$ denotes the velocity dipole matrix element between $\psi_{i}$ and $\psi_{j}$. Explicitly, we use the trajectories calculated from the VASP plane-wave FTDF MD code, developed at the Technical University of Vienna (Kresse \& Hafner 1993); extensive details of this QMD formulation appear elsewhere (Collins et al. 2001).

The KG formulation derives from linear response theory for a system exposed to a weak, external oscillatory electric field and therefore represents a first-order correction to the DFT functions. Quantities such as photoionization and polarizability, calculated in the same manner and employing DFT orbitals, show generally encouraging agreement with experimental measurements for atoms and molecules (Singh \& Deb 1999). A similar finding holds for various optical properties of solids (Cohen \& Chekikowsky 1989). In addition, for warm dense matter conditions, the KG reproduces well both laser and high-energy density experiments for such properties as reflectivity (Collins et al. 2001) and electrical conductivity (Desjarlais et al. 2002). While comprehensive in its range of applications, certain shortcomings such as underestimation of bandgap closings and precise representations of excited states require improvement. Various hybrid and time-dependent DFT methods offer possible systematic cures at the expense of additional computational resources.

The KG formula has the advantage of containing several important limits within its formal structure, for example: 1) at low-frequency to the dc conductivity $\left[\sigma_{1}(\omega) \rightarrow \sigma_{\mathrm{dc}}, \omega \rightarrow 0\right]$; 2 ) at high-frequency to the Kramers formula $\left[\sim 1 / \omega^{3}\right]$; 3) at high-density to the Ziman form; and 4) at low-density to the line spectra from noninteracting atoms or ions. The power of the approach derives from its ability to span accurately the complex regimes between these various limits. This versatility becomes particularly important in representing density effects. For example, many atomic opacity libraries apply the Kramers formula across all photon frequencies. This form however diverges at low-frequencies rather than correctly approaching the finite-valued $\sigma_{\mathrm{dc}}$ of denser systems.

\section{Results}

As an example of the potential of the QMD approach, we consider the deceptively simple system of hydrogen and compare 


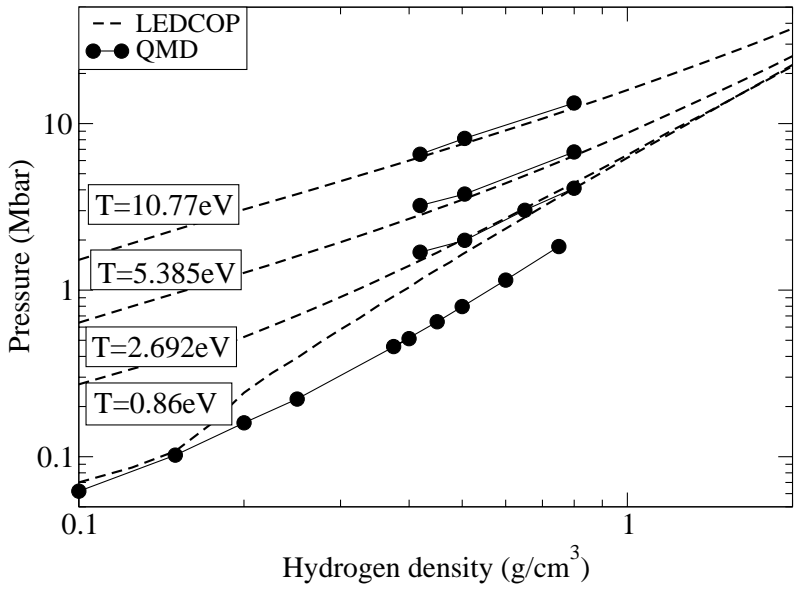

Fig. 1. Comparison pressure as a function of density (EOS) between the LEDCOP (dashed line) and QMD (solid line with dots) for hydrogen at four different temperatures.

to one of the standard opacity libraries, the Los Alamos Light Element Detailed Configuration Opacity Program (LEDCOP) (Magee et al. 1995). LEDCOP provides opacity results (Neuforge-Verheecke et al. 2001) that agree well with other compilations such as OPAL (Iglesias \& Rogers 1996) and CASSANDRA (Crowley \& Harris 2001). We select a representative range $\left[0.1<\rho<1.0 \mathrm{~g} / \mathrm{cm}^{3} ; 5000<T<50000 \mathrm{~K}\right]$ to span a broad regime from high-temperature, low- density, in which QMD and atomic opacity models agree, to high-density, low-temperature for conditions found within the giant planets (Hubbard et al. 2002).

An understanding of the differences in the opacities between the LEDCOP library and the QMD approach requires at first a detailed examination of the underlying material properties such as EOS. The QMD approach, while computationally intense, includes innately transient effects such as dissociation/association of chemical bonds, and ionization/recombination. Consequently, the total pressure obtained from the QMD method reflects not only the constituency of the fluid at a given temperature and density but also various density effects, such as pressure ionization, which are only introduced in a phenomenological fashion in LEDCOP.

In LEDCOP, the EOS model is based on the Saha equation, where the bound Rydberg sequences are cutoff by plasma corrections. The modified Saha equation is solved iteratively to obtain a consistent set of ion abundances, bound state occupancies, and free electrons. In contrast to the QMD simulations, LEDCOP does not account at this point for the possibility of molecular formation.

Figure 1 shows a comparison of the hydrogen isotherms calculated at four different temperatures as obtained with LEDCOP and the QMD simulations. Comparisons along the shock hugoniots with several different experiment and theoretical techniques appear elsewhere (Lenosky et al. 2000; Nellis 2002). At the highest temperature shown, $T=10.77 \mathrm{eV}$ $(125000 \mathrm{~K})$, a reassuring concurrence exists in the predictions of the two methods. At this temperature and for the densities shown, hydrogen is largely a fully ionized plasma, consisting of non-degenerate electrons and protons. As the temperature is lowered (e.g., $2.692 \mathrm{eV}(31250 \mathrm{~K})$ ), the nature of the fluid becomes more complex, consisting of a mixture of atoms, ions, and free electrons. A significant departure between the LEDCOP and QMD results becomes noticeable with significant discrepancy at the lowest temperature, $T=0.86 \mathrm{eV}$ $(10000 \mathrm{~K})$, as the density increases.

Further analysis shows that the disagreement at the lowest temperature comes, at densities greater than $0.1 \mathrm{~g} / \mathrm{cm}^{3}$, from the appearance of molecules, which are not accounted for in LEDCOP. When the density is further increased, the LEDCOP model shows a rapid rise in the total pressure indicating that the atomic fluid rapidly ionizes within a very small density range. This appears as the consequence of the pressure ionization model used that places bound states of radius greater than the ion sphere radius into the continuum. The inadequacy of the model is directly highlighted by the QMD simulation which suggests a smoother increase in the total pressure and thus indicates that the fluid is still not fully ionized up to densities of $1 \mathrm{~g} / \mathrm{cm}^{3}$. Understanding the origins of the discrepancies in the EOS, we now consider the differences in the Rosseland mean opacity.

Figure 2 displays the variation of the Rosseland mean opacity for hydrogen as obtained for fixed density $\left(0.334 \mathrm{~g} / \mathrm{cm}^{3}\right)$ with increasing temperature (a) and for fixed temperature (48000 K) but increasing density (b). For the LEDCOP results, we show both the original calculations, which employ a $n(\omega)$ of unity, and those corrected in Eq. (1) by the index of refraction from the QMD. Some investigators (Perrot 1996; Crowley \& Harris 2001) have suggested that scaling the atomic opacities by an $n(\omega)$ more representative of the medium could approximately introduce some density effects. For low density and a high temperature (Fig. 2a), at which the medium becomes an ionized gas, both versions of LEDCOP and the QMD show good agreement for $\kappa_{R}$. However, as the temperature decreases and the medium becomes only partially ionized, the results begin to diverge. The scaled LEDCOP does follow the QMD results to lower temperature, providing some density correction. However at the lowest temperature, a proper representation of the system, which has evolved into a soup of particles including molecules, requires the fully integrated approach afforded by the QMD. Figure $2 b$ demonstrates that even at a moderately high temperature, for increasing density, collective (many-body) effects play an important role in determining the Rosseland mean opacity.

We further investigate the basic contributions to $\kappa_{R}$ by presenting in Fig. 3 the frequency-dependent absorption coefficient $\alpha(\omega)$ at a high density-temperature point $\left(0.65 \mathrm{~g} / \mathrm{cm}^{3}\right.$, $48000 \mathrm{~K})$ at which the respective equations-of-state are in good agreement. For this case, both LEDCOP and the QMD produce similar Rosseland mean opacities due to the close agreement between the respective absorption coefficients at photon energies around $20 \mathrm{eV}\left(\sim 4 k_{B} T\right)$, the peak of $B^{\prime}$ in Eq. (1) and region of maximum contribution to $\kappa_{R}$. However, the results diverge below and above this energy region. At high photon energies $(\hbar \omega>50 \mathrm{eV})$, the disagreement arises from the finite number of electronic states that can practically be extracted in the FTDF calculation. However, by systematically increasing the number of roots, we can converge to an accurate fit of 

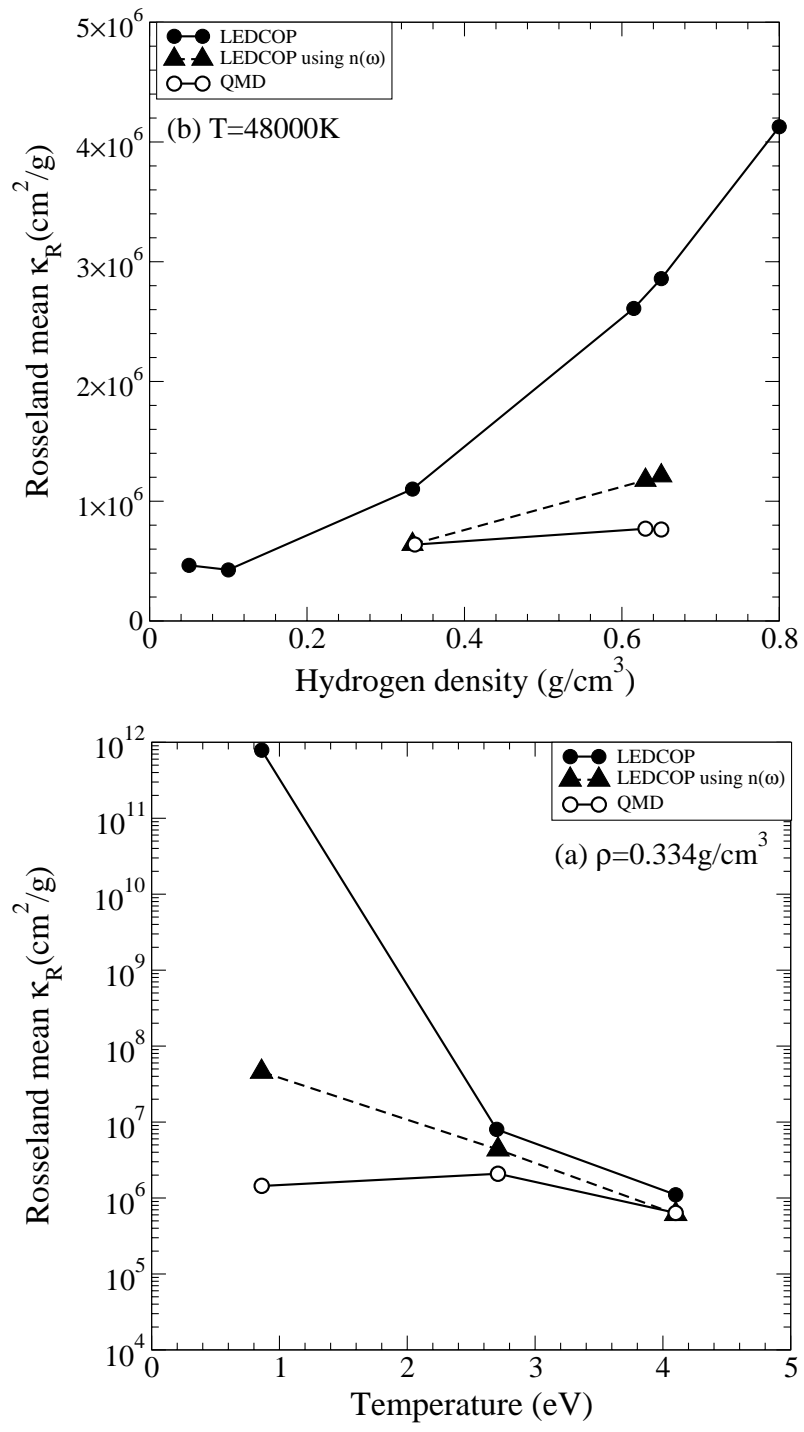

Fig. 2. Variation of the LEDCOP and QMD Rosseland mean opacities a) as a function of temperature and for a fixed density of $\rho=$ $\left.0.334 \mathrm{~g} / \mathrm{cm}^{3}, \mathbf{b}\right)$ as a function of density and for a fixed temperature of $T=48000 \mathrm{~K}$.

the absorption coefficient based on inverse powers of $\hbar \omega$. We have found that such an extrapolation reasonably represents the high-energy behavior of $\alpha(\omega)$ and suffices for the calculation of the Rosseland mean opacity.

For low photon energies, a more substantial disagreement arises. The rapid increase in the LEDCOP absorption coefficient traces directly back to the free-free contribution (inverse Bremsstrahlung) and its $1 / \omega^{3}$ behavior from the Kramers relation. On the other hand, the QMD result declines only slightly as the photon energy decreases. This trend in the QMD stems from the behavior of $\sigma_{1}(\omega)$, which correctly goes to the zero-frequency limit of the DC conductivity $\left(\sigma_{1}(0)=\sigma_{\mathrm{dc}}\right)$. This indicates that hydrogen behaves as a poor metal $\left(\sigma_{\mathrm{dc}} \sim\right.$ $5000 / \mathrm{ohm}-\mathrm{cm})$ in general agreement with the reverberatingshock gas-gun experiments (Weir et al. 1996). Thus, below the plasma frequency $\left(\omega_{\mathrm{p}}\right)$, any electromagnetic wave experiences a strong attenuation, in accordance with the general theory of

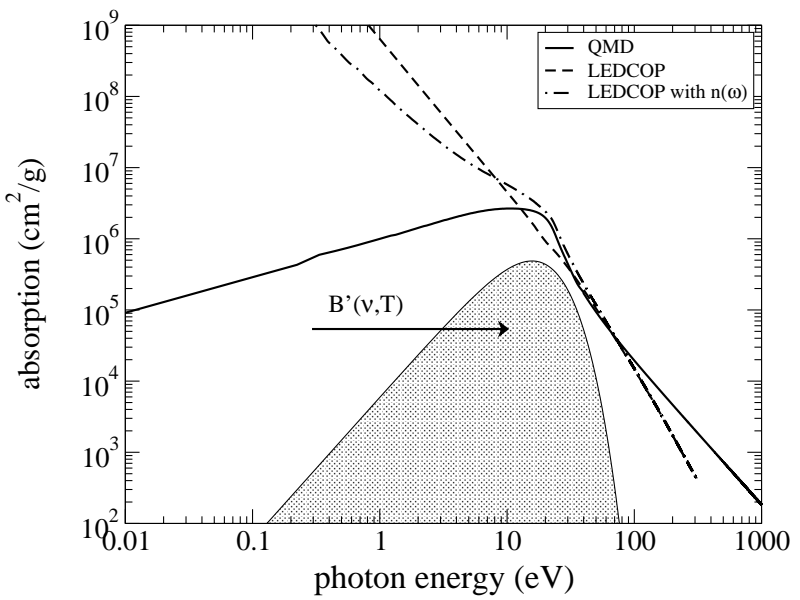

Fig. 3. Variation of the LEDCOP and QMD hydrogen absorption coefficients at a density of $\rho=0.65 \mathrm{~g} / \mathrm{cm}^{3}$ and $T=48000 \mathrm{~K}$. A plot of $B^{\prime}(\omega, T)$ shows the region of maximum contribution to $\kappa_{R}$.

simple metals, as indicated by the QMD $n(\omega)$ increasing to a value of a 100 at $\omega \sim 0$.

LEDCOP modifies the unphysical explosion of the atomic free-free absorption coefficient at low frequencies in the calculation of the Rosseland mean by introducing a step function for the index of refraction $\left[n(\omega)=0, \omega<\omega_{\mathrm{p}} ;=1, \omega \geq \omega_{\mathrm{p}}\right]$. Other investigators (Crowley \& Harris 2001; Iglesias et al. 2002) have offered more systematic corrections based on adjusting the Gaunt factor, for example, with a Drude form for a simple metal. The QMD naturally contains the Drude form when pushed to this limit and, moreover, possesses the capacity to represent the behavior of $\alpha(\omega)$ consistently across a wide range of frequencies for complex media.

The basic qualitative trends found in Fig. 3 for the various absorption coefficients persist over the density-temperature range investigated here; however, the quantitative results can still differ substantially. We can use this basic behavior to understand the differences that arise between LEDCOP and the QMD at lower densities and temperatures. In this case, the region of maximum contribution to $\kappa_{R}$ at around $4 k_{\mathrm{B}} T$ moves to lower photon energies. This exacerbates the large differences in the absorption coefficients in this regime and leads to greater disagreements in the mean opacities as witnessed in Fig. 2.

In conclusion, we have shown that the QMD approach provides a powerful tool to validate and improve models of density effects used in atomic physics approaches within the warm dense matter regime. The current computational capabilities restrict its applicability to the low temperature, high density regime and prevent a complete substitution for standard opacity calculations, especially as the density decreases and the temperature significantly increases from the cases presented here. However, in this low temperature and high density regime where collective effects no longer remain perturbative, the QMD provides an efficient benchmarking tool by establishing a consistent set of material (EOS), dynamical, and optical properties from the same simulation.

Acknowledgements. We acknowledge useful discussions with and suggestions from Drs. W. Huebner and T. Blensky. Work supported 
under the auspices of the U.S. Department of Energy at Los Alamos National Laboratory under Contract W-7405-ENG-36.

\section{References}

Callaway, J. 1974, Quantum Theory of the Solid State (New York: Academic Press)

Chabrier, G., \& Baraffe, I. 2000, ARA\&A, 38, 337

Cohen, M., \& Chelikowsky, J. 1989, Electronic Structure and Optical Properties of Semiconductors, 2nd ed. (Berlin: Springer).

Collins, L., Bickham, S., Kress, J., et al. 2001, Phys. Rev. B, 63, 184110 and references therein

Crowley, B., \& Harris, J. 2001, J. Quant. Spectrosc. Radiat. Transfer, 71,257

Desjarlais, M., Kress, J., \& Collins, L. 2002, Phys. Rev. E, 66, 025401

Fontaine, G., Brassard, P., \& Bergeron, P. 2001, PASP, 113, 409

Hubbard, W., Burrows, A., \& Lunine, J. 2002, ARA\&A, 40, 103

Iglesias, C., \& Rogers, F. 1996, ApJ, 464, 943

Iglesias, C., Rogers, F., \& Saumon, D. 2002 ApJ, 569, L111

Kress, J., Bickham, S., Collins, L., \& Holian, B. 1999, Phys. Rev. Lett., 83, 3896
Kresse, G., \& Hafner, J. 1993, Phys. Rev. B, 47, RC558

Lenosky, T., Bickham, S., Kress, J., \& Collins, L. 2000, Phys. Rev. B, 61,1

Magee, N., Abdallah, J., Clarke, R., et al. 1995, Astron. Soc. Pacific Conf. Proc., 78, 51

Mazevet, S., Johnson, J., Kress, J., Collins, L., \& Blottiau, P. 2002, Phys. Rev. B, 65, 014204

Mazevet, S., Kress, J., Collins, L., \& Blottiau, P. 2003, Phys. Rev. B, 67,054201

Nellis, W. J. 2002, Phys. Rev. Lett., 89, 165502

Neuforge-Verheecke, C., Guzik, J., Keady, J., et al. 2001, ApJ, 561, 450

Perrot, F. 1996, Laser Part. Beams, 14, 731

Rapport, D. C. 1995, The Art of Molecular Dynamics (Cambridge: Cambridge)

Rickert, A. 1995, J. Quant. Spectrosc. Radiat. Transfer, 54, 325

Seaton, M. J., Yan, Y., Mihalas, D., \& Pradhan, A. 1994, MNRAS, 266, 805

Singh, R., \& Deb, B. M. 1999, Phys. Rep., 311, 47 and references therein

Weir, S., Mitchell, A., \& Nellis, W. 1996, Phys. Rev. Lett., 76, 1860 